\title{
A strategy for the reduction of maternal and neonatal mortality
}

Population Council

Follow this and additional works at: https://knowledgecommons.popcouncil.org/departments_sbsr-rh

Part of the International Public Health Commons, Maternal and Child Health Commons, Public Health Education and Promotion Commons, Social and Behavioral Sciences Commons, and the Women's Health Commons How does access to this work benefit you? Let us know!

\section{Recommended Citation}

Population Council. 2010. "A strategy for the reduction of maternal and neonatal mortality," brief. Dakar: Population Council. 


\section{AMTS}

\section{Senegal}

MNCH/FP/MALARIA

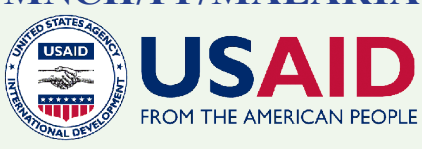

The Situation 1

\section{A strategy for the reduction of maternal and neonatal mortality}

As part of the framework of strategies designed to help reduce maternal and neonatal mortality rates, the introduction of a minimum package of integrated mother and newborn health care, including the Active Management of Third Stage Labor (AMTSL or "GATPA" in French) and the administration of Essential Newborn Care (ENC), constitutes a simple and inexpensive approach for poor countries.

\section{Context}

The rate of maternal mortality is 401 maternal deaths for every 100,000 live births (EDS/Sénégal, 2005). The immediate post-partum period is critical for both mother and newborn, given that approximately $60 \%$ of maternal deaths and around $75 \%$ of newborn deaths occur in the first week following delivery (Lancet, 2006). As part of a framework of strategies designed to reduce maternal and neonatal mortality, the introduction of a minimum package of integrated health care for the mother and infant, including the Active Management of Third Stage Labor (AMTSL, GATPA in French) and the administration of Essential Newborn Care (ENC, SENN in French), serves as a simple and inexpensive approach within poor countries.

The Ministry of Health and Prevention (MSP), through the Division of Reproductive Health (DSR) and with the support of IntraHealth International, initiated an important program aimed at strengthening the capacities of service providers in AMTSL and ENC at the regional level, in Louga, Thies, Kaolack, Kolda and Ziguinchor regions, where the integrated packet of Maternal, Child, and Infant Health/FP/Malaria (SMNI/
$\mathrm{PF} /$ Paludisme in French) was being implemented.

A study was conducted with the following objectives: 1) evaluate the functional capacity of the targeted health facilities to offer AMTSL and ENC; 2) evaluate the knowledge and competence of service providers trained in AMTSL and ENC;

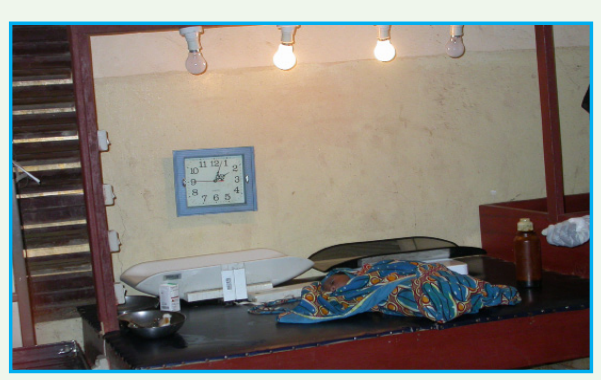
3) identify problems encountered during the implementation of AMTSL and $\mathrm{ENC}$; and 4) formulate recommendations. This study included 170 service providers at 98 health centers (PPS) in the 26 health districts of the five targeted regions where service providers benefited from AMTSL and ENC training. The functional capacity of the facilities to offer AMTSL and ENC as well as the performance of the service providers were measured according to a scoring system that placed the acceptable level of provider adherence at $100 \%$ for knowledge and $85 \%$ for competence.

\section{Results}

\section{Work environment}

The availability of infrastructure and necessary basic materials (such as separate areas for newborns, waste disposal, refrigerators) in the delivery wards were 
lacking at the sites visited with the average infrastructure score of 58 points. The average score for the availability of newborn care supplies was low (51 points), as was the score for available infection prevention materials (53 points).

Poor availability of the medicines and products for AMTSL was observed with an average score of 53 points. ENC medicines for newborns were more available but the average score was still fairly low (69 points). It was observed that certain products such as Misoprostol and Vitamin $\mathrm{K}$ were particularly lacking, and problems with the product preservation were revealed, such as Oxytocin which was not stored according to protocol in $30 \%$ of facilities.

\section{What is the level of procedural knowledge?}

The data indicate that service providers have knowledge of AMTSL procedures (93.3\%). As for ENC, the theoretical knowledge is very satisfactory with 8 out of 10 service providers able to cite the five elements of basic newborn care.

\section{What is the level of actual practice?}

Based on the sample of 170 cases, observation of this practice has shown that the three key elements in the AMTSL are well followed by the service providers. However, actions should be taken to improve adherence to certain steps that are no less significant, notably that of preparation, where the score remains low.

\section{Average performance score of AMTSL procedures by service provider}

\begin{tabular}{|l|l|}
\hline Preparation score & $56 \%$ \\
\hline Delivery score & $83 \%$ \\
\hline Uterine massage score & $66 \%$ \\
\hline Basic newborn care score & $79 \%$ \\
\hline Data recording score & $73 \%$ \\
\hline
\end{tabular}

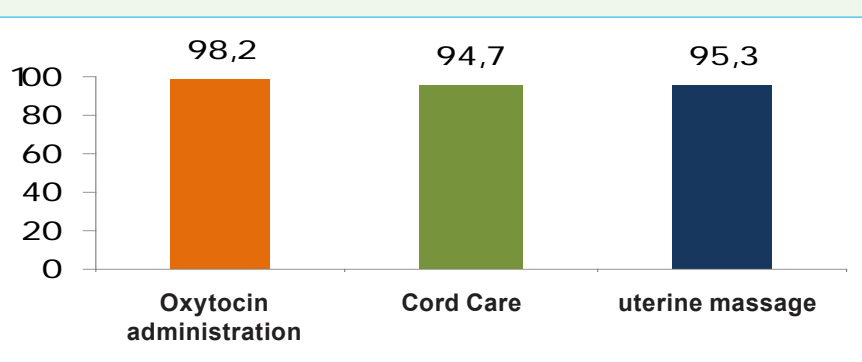

The results show an equal satisfaction of adherence to following the five key elements of the Essential Newborn Care procedures after delivery.

The performance analysis reveals dysfunctions in the work environments of the service providers. An insufficiency and lack of both AMTSL and ENC products have been noted at the facility level. Only $29.6 \%$ of service providers stated they have all the material necessary to provide newborn care at their disposal. Only $41 \%$ of service providers were supervised by a superior during the last three months and among those, only $77 \%$ received immediate feedback on their performance.

\begin{tabular}{|l|r|}
\hline $\begin{array}{l}\text { Percentage of service providers who follow the } \\
\text { key elements of ENC procedures }\end{array}$ \\
\hline Maintain temperature & $87 \%$ \\
\hline Immediate breastfeeding & $92 \%$ \\
\hline Clean cord care & $81 \%$ \\
\hline Eye drop administration & $92 \%$ \\
\hline Vitamin K administration & $69 \%$ \\
\hline
\end{tabular}

\section{Recommendations}

- Improve the work environment of service providers to strengthen practices at the site level;

- Increase the availability of products for AMTSL and ENC and improve the input storage conditions;

- Promote greater supervision to sustain acquired knowledge and skills;

- Focus on data reporting through systematic registration and methods to facilitate documentation

This document is made possible by the generous support of the american people through the United States Agency for International Development (USAID). The contents are the responsibility of the authors and do not reflect the views of USAID or the United States Government. 